\title{
Statin therapy in patients with community-acquired pneumonia
}

\author{
Authors: Frances S Grudzinska, ${ }^{\mathrm{A}}$ Davinder PS Dosanjh, ${ }^{\mathrm{B}}$ Dhruv Parekh, ${ }^{\mathrm{C}}$ Rachel CA Dancer, ${ }^{\mathrm{B}}$ Jaimin Patel, ${ }^{\mathrm{D}}$ \\ Peter Nightingale, ${ }^{\mathrm{E}}$ Georgia M Walton, ${ }^{\mathrm{F}}$ Elizabeth Sapey ${ }^{\mathrm{G}, *}$ and David R Thickett ${ }^{\mathrm{H}, *}$
}

\begin{abstract}
Community-acquired pneumonia (CAP) is the leading cause of death from infection in developed countries. There is evidence of an association between improved survival from infection and statin use. The possible beneficial effects of statins are complicated by the common use of macrolide antibiotics for pneumonia, with current guidance suggesting that concurrent macrolide and statin use is contraindicated. We conducted an observational study of statin use in patients with CAP. Of 2,067 patients with CAP, $30.4 \%$ were on statin therapy at admission. Statin users were more likely to survive the admission $(p<0.001)$. In addition, we conducted a survey of doctors and found that knowledge regarding concurrent macrolide and statin use was lacking. These data suggest a potential role of statins in the management of CAP. Further research using high-dose statins is required to assess their safe use in subjects with mild to moderate infections.
\end{abstract}

KEYWORDS: Immunomodulatory drugs, macrolide antibiotic, pneumonia, sepsis, statin

\section{Introduction}

Pneumonia is the leading cause of infectious death in developed countries, and mortality rates have not improved in the last decade. ${ }^{1}$ Patients admitted to hospital with community-acquired pneumonia (CAP) have approximately $20 \%$ mortality, rising to $35 \%$ if they develop sepsis and

Authors: ${ }^{\text {A }}$ academic clinical fellow in respiratory medicine, Institute of Inflammation and Ageing, University of Birmingham, Birmingham, UK; ${ }^{B}$ lecturer in respiratory medicine, Institute of Inflammation and Ageing, University of Birmingham, Birmingham, UK; ${ }^{C}$ clinician scientist in respiratory and critical care medicine, Institute of Inflammation and Ageing, University of Birmingham, Birmingham, UK; ${ }^{\mathrm{D}}$ Clinician scientist in critical care medicine, Institute of Inflammation and Ageing, University of Birmingham, Birmingham, UK; ${ }^{E}$ statistician, University Hospital Birmingham NHS Foundation Trust, Birmingham, UK; 'F research associate, Institute of Inflammation and Ageing, University of Birmingham, Birmingham, $U K$; ${ }^{G}$ reader in respiratory medicine, Institute of Inflammation and Ageing, University of Birmingham, Birmingham, UK; ${ }^{\mathrm{H}}$ chair of respiratory medicine, Institute of Inflammation and Ageing, University of Birmingham, Birmingham, UK; *authors contributed equally
$45 \%$ if they develop multi-organ failure. ${ }^{2}$ It is predicted that hospitalisation for CAP may double by $2040 .^{3}$

Although inflammation is a necessary physiological response to infection, it has been suggested that an excessive and dysregulated inflammatory response contributes to the pathology and hence morbidity and mortality seen in CAP. ${ }^{4}$ Multiple trials have attempted to modulate this excessive immune response using corticosteroids and other drugs, but none have demonstrated significant benefit. ${ }^{5}$ With a dearth of novel drug development for infection in recent years, increasingly efforts have focused on repurposing established drugs and investigating their possible immunomodulatory effects.

HMG-CoA reductase inhibitors (statins) competitively inhibit HMG-CoA reductase, the first enzyme active in the mevalonate pathway, and are the most commonly prescribed drugs in the UK. ${ }^{6}$ They are conventionally used as cholesterol lowering agents; however, a number of population studies and clinical trials have suggested survival benefits during infection ${ }^{7-10}$ and pre-dosing with statins improves outcomes in murine sepsis models. ${ }^{11}$ Inhibition of the mevalonate pathway results in reduced production of isoprenoids, which are required for the function of small GTP-ases and therefore essential for innate and adaptive immunity pathways. ${ }^{12-14}$ Suggested beneficial effects of statins include improved neutrophil function, ${ }^{15,16}$ decreased inflammatory cell influx into the lungs, ${ }^{17}$ reduced pro-inflammatory cytokine release, ${ }^{17,18}$ prevention of T-cell activation $^{19}$ and reduction of local and systemic levels of C-reactive protein (CRP). ${ }^{20}$ Based on this, preclinical and observational studies have been performed and suggest a benefit in those with infection; however, these findings have not translated to positive outcomes in randomised controlled trials (RCTs) of established severe sepsis or acute respiratory distress syndrome. The reasons for this remain unclear.

There is no clear guidance on whether statins should be continued on admission to hospital with infection. A single study examined the benefits of continued statin therapy or placebo in critically ill patients and found no difference in survival or progression of sepsis. ${ }^{21}$

The National Institute for Health and Care Excellence (NICE) guidance suggests that all patients with either moderate or severe CAP should receive a macrolide antibiotic in addition to either amoxicillin or a beta-lactam antibiotic. ${ }^{22}$ Commonly, clarithromycin is used as the macrolide component, which 
is a potent enzyme inhibitor. Medicines and Healthcare products Regulatory Agency (MHRA) guidance suggests statins should not be concurrently administered with clarithromycin because of the risk of rhabdomyolysis and acute renal failure. ${ }^{23}$ The absolute risk of acute kidney injury caused by statin and clarithromycin co-treatment was $0.02 \%$, although this represents a relative risk of 2.06 compared with concurrent statin and azithromycin treatment. ${ }^{24}$ In UK hospital practice, macrolide-statin co-prescription is monitored as an indicator of prescribing safety and as a result a large proportion of patients have statin therapy paused on admission. ${ }^{25}$ With conflicting data in the literature and the issues surrounding macrolidestatin co-prescription, it remains unclear whether statins are of benefit to those with CAP.

We therefore undertook a retrospective study to assess the association of statin therapy at hospital admission with clinical outcomes for CAP. In addition, knowledge of macrolide-statin co-prescription guidance among the consultant and traininggrade doctors in a large teaching hospital was evaluated.

\section{Methods}

\section{Study institution and CAP subjects}

All adults admitted with CAP to the University Hospitals Birmingham NHS Foundation Trust (UHB) between October 2009 and November 2011 were eligible for inclusion. These patients were identified using the hospital's coding system, using pre-defined ICD-10 codes (J12-16, 18). UHB is a large, tertiary centre teaching hospital in the West Midlands, UK, which admits unselected adults for acute medical care.

CAP was defined as an acute lower respiratory tract illness associated with new radiographic shadowing for which there was no other explanation, in accordance with British Thoracic Society guidelines. ${ }^{26}$ The radiographs and clinical notes of all patients eligible for inclusion underwent clinical review to confirm the diagnosis. As a marker of CAP severity, the CURB-65 score was calculated. Using electronic patient records the following data were captured: age, gender, diagnosis of diabetes mellitus, length of hospital admission, intensive care unit (ICU) admission, ICU length of stay, CRP, white cell count (WCC), urea and statin use (defined as a statin prescription within 24 hours of admission). The primary outcome measure was inpatient mortality. Secondary outcome measures included length of hospital stay, ICU admission and ICU length of stay.

\section{Macrolide-statin co-prescription surveys}

We devised an online survey to evaluate the knowledge of consultant and training-grade doctors working at the same trust the study was conducted in (UHB). The survey was designed using Survey Monkey ${ }^{\mathrm{TM}}$ (Oregon, USA) and emailed to all doctors attending the Clinical Grand Round in November 2016.

The survey asked the following four questions:

1 What grade are you? (Junior doctor/Consultant)

2 Are you aware of any drug interactions with clarithromycin? (Yes/No)

3 Are you aware of an interaction between statins and clarithromycin? (Yes/No)

4 Do you pause statins when prescribing clarithromycin? (Yes/No/Sometimes)
Patients coded as CAP between October 2009 and November 2011 $n=2,225$

Clinical diagnosis of bacterial or unspecified pneumonia $n=2,067$

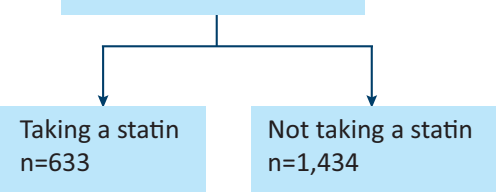

Fig 1. Modified CONSORT diagram demonstrating patient inclusion pathways and reasons for exclusion. BTS = British Thoracic Society; $\mathrm{CAP}=$ community-acquired pneumonia; $\mathrm{CONSORT}=$ Consolidated Standards of Reporting Trials.

\section{Statistical analysis}

Normality was assessed using the Kologmorov-Smirnov test. Statin-users and non-users were compared using the MannWhitney-U test when variables were continuous and $\chi^{2}$-test for categorical variables. Logistic regression was used to calculate a predicted probability of death and evaluate the effects of statin therapy on the predicted survival from pneumonia. This was done using a model taking into account age and statin therapy in the first instance. This model was then refined to correct for gender, diabetic status and CRP. Significance was accepted at $\mathrm{p}<0.05$. Survey data were analysed using a Chi-squared test. Statistical analyses were carried out using PASW v18.0 (Chicago, IL, USA).

\section{Results}

A total of 2,225 patients were identified as having CAP by the hospital coding system. In total, 2,067 patient episodes were included for final analysis; of these, 633 were taking a statin on admission and 1,434 were not (Fig 1). Simvastatin was the most commonly used statin $(75.5 \%)$, followed by atorvastatin (19.4\%), fluvastatin (4.7\%) and rosuvastatin (0.2\%).

\section{Demographics and clinical characteristics of patients}

Approximately half of patients were male (50.6\%), with a mean age (SD) of 71.2 years (18.2). Statin users were older (statin user versus non-user: mean \pm SD $75.6 \pm 11.7$ years versus $69.2 \pm 20.1$ years, $\mathrm{p}<0.001$ ) and had a higher prevalence of diabetes (statin user versus non-user: $36.4 \%$ versus $12.1 \%, \mathrm{p}<0.001$ ). Statin 
Table 1. Cohort characteristics

\begin{tabular}{|c|c|c|c|c|}
\hline & Statin user $n=633$ & Non-user $n=1,434$ & Total, n (\%) & p-value \\
\hline $\mathrm{n}(\%)$ & $633(30.7)$ & $1,434(69.3)$ & 2,067 & \\
\hline Mean age $\pm S D$, years & $75.66 \pm 11.7$ & $69.2 \pm 20.1$ & & $<0.001$ \\
\hline \multicolumn{5}{|l|}{ Gender, n (\%) } \\
\hline Male & $355(56.2)$ & $691(48.2)$ & $1,046(50.6)$ & 0.001 \\
\hline Female & $278(43.8)$ & $743(51.8)$ & $1,021(49.4)$ & \\
\hline \multicolumn{5}{|l|}{ Patient outcome, n (\%) } \\
\hline Survived & $526(83.1)$ & $1,083(75.5)$ & $1,609(77.9)$ & $<0.001$ \\
\hline Died & $107(16.9)$ & $351(24.5)$ & $458(22.1)$ & \\
\hline \multicolumn{5}{|l|}{ Diabetes status, n (\%) } \\
\hline Yes & $230(36.4)$ & $174(12.1)$ & $404(19.6)$ & $<0.001$ \\
\hline No & $403(63.6)$ & $1,260(87.9)$ & $1,663(80.4)$ & \\
\hline Mean length of stay $\pm S D$, days & $13.5 \pm 15.2$ & $8.7 \pm 12.7$ & & $<0.001$ \\
\hline \multicolumn{5}{|l|}{ ICU admission, n (\%) } \\
\hline Yes & $67(10.6)$ & $102(7.1)$ & $169(8.1)$ & 0.008 \\
\hline No & $567(89.4)$ & $1,331(92.9)$ & $1,898(91.9)$ & \\
\hline Mean ICU length of stay $\pm S D$, days & $8.6 \pm 10.3$ & $7.7 \pm 8.9$ & $169(8.1)$ & 0.483 \\
\hline Median CURB-65 score (IQR) & $2(2-2)$ & $2(1-3)$ & $2,006(97)$ & $<0.001$ \\
\hline Median CRP median, mg/L (IQR) & $93(34-211)$ & $102(43-215)$ & $1,801(87)$ & 0.188 \\
\hline Median WCC, $\times 10^{9} / L$ (IQR) & $12.8(9.3-16.5)$ & $12.6(8.9-16.9)$ & $1,573(76)$ & 0.709 \\
\hline Median urea, mmol/L (IQR) & $8.6(6.1-12.3)$ & $7.3(4.9-11.3)$ & $2,006(97)$ & $<0.001$ \\
\hline
\end{tabular}

users had higher urea $(\mathrm{p} \leq 0.005)$ and were more likely to be male $(\mathrm{p}<0.001)$ (Table 1$)$.

\section{In-hospital mortality}

In terms of in-hospital mortality, survival was significantly higher among statin users (statin user $83.1 \%$ versus non-user $75.5 \%, \mathrm{p}<0.0001)$.

Using logistic regression, predicted probability of in-hospital death was calculated, while controlling for age and gender (Fig 2). The odds ratio (OR) for in-hospital death from pneumonia was 1.046 ( $\mathrm{p}<0.001,95 \%$ CI 1.038-1.054) for each additional year

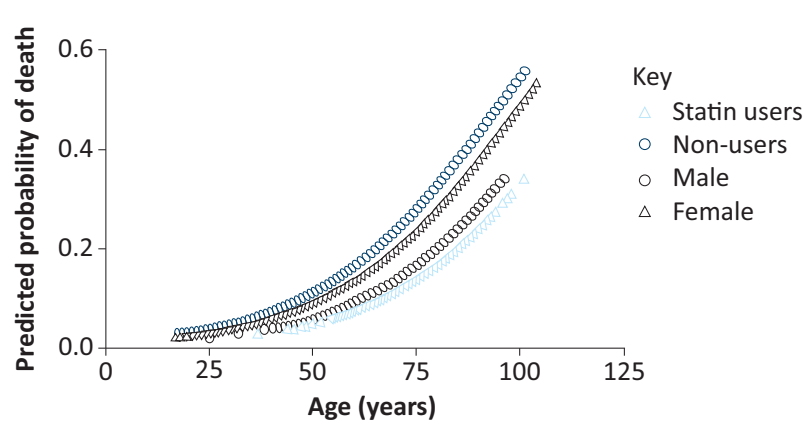

Fig 2. Relationship between predicted probability and death and increasing age. Stratified according to statin users and non-users, and gender. of age. The OR for in-hospital death was higher in males (1.249, $\mathrm{p}<0.001$, CI 1.003-1.556). When taking a statin, the OR for inhospital death was reduced to 0.515 ( $\mathrm{p}<0.001$, CI $0.403-0.660$ ).

After adjusting for disease severity (as assessed by CURB65 score), age, diabetes and CRP, outcomes were better in patients on higher doses of simvastatin (Table 2). There was no statistically significant benefit, in terms of OR for inhospital mortality, in those who received a simvastatin dose above $40 \mathrm{mg}$. The class of statin administered had no effect on mortality (data not shown).

\section{Secondary outcome measures}

Statin users had increased length of hospital stay (statin user versus non-user 13.5 days versus 8.7 days, $\mathrm{p}<0.001$ ) and were more likely to be admitted to the ICU (statin user $10.6 \%$ versus non-user $7.1 \%, \mathrm{p}=0.008$ ). Although there was a statistically significant difference in CURB-65 score, the two groups were clinically comparable. There was no difference in WCC or CRP between the two groups (Table 1).

\section{Survey of knowledge regarding statin and macrolide antibiotics}

One hundred responses were submitted using the free online survey software; 61 consultants (61\%) and 39 trainees (39\%). Of those who responded to the survey, $84 \%$ were aware of drug interactions with clarithromycin and $72 \%$ were aware of the 
Table 2. Relationship between simvastatin dose and mortality

n

Simvastatin dose

$10 \mathrm{mg}$

$20 \mathrm{mg}$

$40 \mathrm{mg}$

$80 \mathrm{mg}$

36
B 15 76 4.346

3.019 352
SE

OR

(95\% CI)

p-value

Relationship assessed by logistic regression and adjusted for age and disease severity (CURB-65 score). To assess the effect on mortality of increasing doses of simvastatin, patients taking $10 \mathrm{mg}, 20 \mathrm{mg}$ and $80 \mathrm{mg}$ were compared to those on $40 \mathrm{mg}$.

$\mathrm{B}=$ regression coefficient; $\mathrm{CI}=$ confidence interval; $\mathrm{OR}=$ odds ratio; $\mathrm{SE}=$ standard error specific interaction between statins and clarithromycin. When asked if they pause statin therapy for the duration of macrolide treatment responses varied with $40 \%$ responding 'yes', $31 \%$ 'no' and $29 \%$ 'sometimes'.

When stratified according to training grade, trainee doctors were more likely to be aware of the drug interactions than consultants $(\mathrm{p}=0.039)$ and were more likely to always pause statin therapy when prescribing a macrolide antibiotic $(p=0.02)$.

\section{Discussion}

Our data suggest that patients with CAP who are taking a statin on admission have reduced risk of in-patient mortality. This is despite being older, having increased length of hospital stay and increased likelihood of requiring ICU admission.

Despite clear MHRA guidance regarding concurrent statin and macrolide prescription, we have demonstrated that knowledge and practice in this area is lacking. With increasing numbers of patients expected to start taking statins, this is set to become a more frequent clinical issue.

The mechanism by which statins reduce risk of in-patient death in this cohort remains unclear. Statins influence multiple anti-inflammatory pathways; ${ }^{12-14}$ it may be that stopping statin therapy at a time of hyper-inflammation could cause rebound inflammation and adversely affect patient outcomes. There is also evidence to suggest that statins are able to modulate immune cell function, which could reduce the damaging inflammation associated with organ dysfunction. Alternatively, it is estimated that cardiovascular events account for up to $30 \%$ of the mortality associated with CAP, and hence the beneficial effect we have observed could be directly related to the lipidlowering effects and plaque stabilisation. ${ }^{27}$

Despite promising observational studies and animal model data, no RCT to date has demonstrated a beneficial effect from statin therapy in established sepsis. There is no consensus as to why this may be. In vitro cellular studies often utilise statin concentrations that exceed standard prescribing regimens while RCTs have often used low-dose statins. ${ }^{28}$ In addition, RCTs are often carried out in patients of varying age with established sepsis on short-term therapy while population studies have included older patients who were taking statins prior to the infective event, as is the case in our study. ${ }^{28}$ This may suggest that timing of statin therapy is important or that statins are not beneficial at the stage of severe sepsis but may have greater utility in mild infection ${ }^{29}$ and in preventing progression to severe sepsis. In keeping with this, atorvastatin has been shown to prevent the progression of mild ward-based sepsis patients to severe sepsis in an interventional trial. ${ }^{30}$

It may also be the case that statins are not an effective therapy for all septic patients and further work needs to be completed to identify patient groups who would benefit from this potentially immunomodulatory therapy. Furthermore, the dose of statin used in the RCTs has been heterogeneous; it may be that high doses of statins are required prior to the onset of severe sepsis. In the current retrospective study, the higher doses of statins were associated with the greatest in hospital survival benefit.

In the UK, admission to the ICU is dependent on severity of the acute illness, identification of a reversible cause, as well as the physiological reserve of the patient prior to illness onset. Increased rates of ICU admission among statin users in this study may seem to suggest increased severity of pneumonia and sepsis. However, there was no clinical difference in the severity of pneumonia between statin using and non-using adults in the current study and therefore it may also be the case that statin users have greater physiological reserve and hence are more likely to be accepted for ICU admission.

Length of hospital stay is also related to severity of illness, physiological capacity for recovery as well as provision of social support on discharge. The increased length of stay among statin users may again indicate that these patients either had more severe pneumonia (which we did not find) or a prolonged recovery period. Our analysis included the length of stay of all patients, whether they died in hospital or not; those who survived may have had longer hospital stays.

Statins are commonly prescribed at present but are set to become almost ubiquitous given new NICE guidance. ${ }^{31}$ Our data suggest that statins are beneficial in CAP, but the risk versus benefit ratio in co-prescription of statins and macrolides may need to be evaluated.

Our study has limitations; it is a retrospective design, which limits our ability to account for confounders, although we have demonstrated that the severity of pneumonia was similar across the two groups. We cannot demonstrate causality and healthcare-seeking behaviours must be considered as an important confounding factor. The timing of statin administration and dose of statin seems to be crucial in their beneficial effect as demonstrated by the divergent evidence from existing population studies, RCTs and this study. We used the hospital's electronic patient record for mortality data, which has inaccuracies in outpatient mortality as well as cause of death data. Given this, we reported inpatient death and allcause mortality as outcomes, which may have introduced bias. 


\section{Conclusions}

Statin use at the time of hospital admission is associated with lower in-hospital mortality in CAP, despite older age of patients and higher burden of diabetes. RCTs are yet to show conclusive benefits of statins in sepsis; this may be due to the timing of statin administration, dose heterogeneity or statins being ineffective once sepsis is established.

Further RCTs of statins in mild to moderate infections are warranted, with particular efforts to identify patient characteristics linked to benefit from immunomodulatory therapy.

\section{Conflicts of interest}

The authors have no conflicts of interest to declare.

\section{Author contributions}

FSG performed data acquisition, analysis and interpretation for the article. DPSD, DP, RCAD and JP helped with data interpretation. PN performed the statistical analysis of the data. GW helped collate clinical data. ES confirmed diagnosis of CAP and both ES and DT designed the study. All authors were involved with drafting and revising the work and approved the final submission.

\section{Acknowledgments}

We are grateful to the Bio-Informatics Team at UHB for their help in extracting data and the nursing team at the NIHR Clinical Research Facility for their help in collating clinical data.

\section{References}

1 Lindenauer PK, Lagu T, Shieh M, Pekow PS, Rothberg MB. Association of diagnostic coding with trends in hospitalizations and mortality of patients with pneumonia, 2003-2009. JAMA 2012;307:1405-13.

2 Dellinger RP, Carlet JM, Masur H et al. Surviving Sepsis Campaign guidelines for management of severe sepsis and septic shock. Crit Care Med 2004;32:858-73.

3 Wroe PC, Finkelstein JA, Ray GT et al. Aging population and future burden of pneumococcal pneumonia in the United States. J Infect Dis 2012;205:1589-92.

4 Viasus D, Garcia-Vidal C, Simonetti AF et al. The effect of simvastatin on inflammatory cytokines in community-acquired pneumonia: a randomised, double-blind, placebo-controlled trial. BMJ Open 2015;5:e006251.

5 Meijvis SC, van de Garde EM, Rijkers GT, Bos WJ. Treatment with anti-inflammatory drugs in community-acquired pneumonia. J Intern Med 2012;272:25-35.

6 Scholes S, Faulding S, Mindell J. Use of prescribed medicines. In: Health Survey for England - 2013. Leeds: HSCIC, 2014: chapter 5.

7 Bjorkhem-Bergman L, Bergman P, Andersson J, Lindh JD. Statin treatment and mortality in bacterial infections-a systematic review and meta-analysis. PLoS One 2010;5:e10702.

8 Janda S, Young A, Fitzgerald JM, Etminan M, Swiston J. The effect of statins on mortality from severe infections and sepsis: a systematic review and meta-analysis. J Crit Care 2010;25:656.e7-22.

9 Chopra V, Rogers MA, Buist M et al. Is statin use associated with reduced mortality after pneumonia? A systematic review and meta-analysis. Am J Med 2012;125:1111-23.

10 Khan AR, Riaz M, Bin Abdulhak AA et al. The role of statins in prevention and treatment of community acquired pneumonia: a systematic review and meta-analysis. PLoS One 2013;8:e52929.

11 Catron DM, Lange Y, Borensztajn J et al. Salmonella enterica serovar Typhimurium requires nonsterol precurosers of the cholesterol biosynthetic pathway for intracellular proliferation. Infect Immun 2004;72:1036-42.
12 Kinsella A, Raza A, Kennedy S et al. The impact of high-dose statin therapy on transendothelial neutrophil migration and serum cholesterol levels in healthy male volunteers. Eur J Clin Pharmacol 2011;67:1103-8.

13 Al-Ani B. Simvastatin inhibits neutrophil degranulation induced by anti-neutrophil cytoplasm auto antibodies and N-fMLP. Saudi Med J2013;34:477-83.

14 Silveira A, Dominical V, Lazarini M, Costa F, Conran N. Simvastatin abrogates inflamed neutrophil adhesive properties, in association with the inhibition of Mac-1 integrin expression and modulation of Rho kinase activity. Inflamm Res 2013;62:127-32.

15 Chow OA, von Kockritz-Blickwede M, Taylor Bright A et al. Statins enhance formation of phaogocyte extracellular traps. Cell Host and Microbe 2010;8:445-54.

16 Walton GM, Stockley JA, Griffiths D et al. Repurposing treatments to enhance innate immunity. Can statins improve neutrophil functions and clinical outcomes in COPD? J Clin Med 2016;5:E89.

17 Shyamsundar M, McKeown ST, O'Kane CM et al. Simvastatin decreases lipopolysaccharide-induced pulmonary inflammation in healthy volunteers. Am J Respir Crit Care Med 2009;179:1107-14.

18 Iwata A, Shirai R, Ishii $\mathrm{H}$ et al. Inhibitory effect of statins on inflammatory cytokine production from human bronchial epithelial cells. Clin Exp Immunol 2012;168:234-40.

19 Kwak B, Mulhaupt F, Myit S, Mach F. Statins as a newly recognized type of immunomodulator. Nat Med 2000;6:1399-402.

20 McDowell SA, Ma Y, Kusano R, Akinbi HT. Simvastatin is protective during Staphylococcus aureus pneumonia. Curr Pharm Biotechnol 2011;12:1455-62.

21 Kruger PS, Harward ML, Jones MA et al. Continuation of statin therapy in patients with presumed infection: a randomized controlled trial. Am J Respir Crit Care Med 2011;183:774-81.

22 National Institute for Health and Care Excellence. Pneumonia in adults: diagnosis and management. NICE clinical guideline No 191. London: NICE, 2014.

23 Medicines and Healthcare producs Regulatory Agency. Simvastatin: updated advice on drug interactions. London: MHRA, 2012. www.gov.uk/drug-safety-update/simvastatin-updated-advice-ondrug-interactions [Accessed 4 July 2017].

24 Patel AM, Shariff S, Bailey DG et al. Statin toxicity from macrolide antibiotic coprescription: a population-based cohort study. Ann Intern Med 2013;158:869-76.

25 Thomas SK, McDowell SE, Hodson J et al. Developing consensus on hospital prescribing indicators of potential harms amenable to decision support. Br J Clin Pharmacol 2013;76:797-809.

26 Lim WS, Baudouin SV, George RC et al. BTS guidelines for the management of community acquired pneumonia in adults: update Hennessy E, Adams C, Reen FJ, O'Gara F. Is there potential for repurposing statins as novel antimicrobials? Antimicrob Agents Chemother 2016;60:5111-21.

28 Patel J, Thickett DR, Gao F, Sapey E. Statins for sepsis: distinguishing signal from the noise when designing clinical trials. Am J Respir Crit Care Med 2013;188:874.

29 Jia M, Huang W, Li L et al Statins Reduce mortality after nonsevere but not after severe pneumonia: a systematic review and meta-analysis. J Pharm Pharm Sci 2015;18:286-302.

30 Patel JM, Scaith C, Thickett D et al. Atorvastatin for preventing the progression of sepsis to severe sepsis (ASEPSIS Trial): a randomised, double-blind, controlled trial. Crit Care Med 2011;15:268.

31 National Institute for Health and Care Excellence. Cardiovascular disease: risk assessment and reduction, including lipid modification. NIC clinical guideline No 181. London: NICE, 2016.

Address for correspondence: Professor David Thickett, Institute of Inflammation and Ageing, University of Birmingham, Edgbaston, Birmingham B15 2TT, UK. Email: D.thickett@bham.ac.uk 